\title{
CORPORALIDADES, TEMPORALIDADES E INFANCIAS: LA PREGUNTA POR LA HOSPITALIDAD DESDE LA ESCUELA
}

Malvina Argumedo ${ }^{l}$

\section{CONSIDERACIONES INICIALES}

Las escuelas y aulas de la Modalidad de Educación Hospitalaria son formas organizativas de la educación primaria que funcionan en hospitales pediátricos, para permitir la continuidad escolar de niños y niñas que se encuentran atravesando una situación de enfermedad y permanecen internados en dichos establecimientos. Esta propuesta educativa tiene un gran valor como derecho conquistado en el camino para que todo niño y niña, cualquiera sea su situación, tenga acceso a una educación que lo reconozca como sujeto, que le brinde las oportunidades de participar de un proyecto cultural y social común y amplio desde su singularidad, desde su voz y su mirada.

Transitar por estas escuelas y por las experiencias de sus protagonistas, nos invita a preguntarnos en torno a los sentidos que la propuesta educativa asume en relación a una infancia que padece, que sufre, cuya corporalidad se vuelve presencia plena y su subjetividad deviene entre prescripciones, intervenciones y resistencias.

Mientras que hoy con el adjetivo hospitalario la medicina califica a un establecimiento, un ambiente destinado al diagnóstico y tratamiento de individuos "enfermos" que son "alojados" temporalmente allí para recibir una intervención sobre su cuerpo, para la recuperación de sus funciones orgánicas y bienestar somático, pensar desde la educación la hospitalidad y lo hospitalario como características de una escuela, excediendo la referencia directa a un contexto particular de trabajo, puede darnos otras claves para recrear los sentidos de lo educativo en estos espacios, para preguntarnos por los modos en que en ellos son recibidas las infancias y pensar su potencia como posibles lugares de experiencias transformadoras para sus protagonistas.

\footnotetext{
${ }^{1}$ CONICET-FLACSO/UNLP malvinaargumedo@gmail.com
} 


\section{LA CUESTIÓN DE LA ENUNCIACIÓN Y LAS LÓGICAS DE CONTROL EN DISCURSOS Y PRÁCTICAS MÉDICO-PEDAGÓGICAS}

La educación, por medio del dispositivo escolar, y la medicina, mediante su práctica actual en los hospitales, han ejercido un poder y construido un saber que ha cristalizado en un discurso cuya lógica está destinada a nombrar, demarcar, clasificar y disciplinar cuerpos y subjetividades. Centramos en el discurso hegemónico de estas dos instituciones la idea de "normalidad", que funciona como eje común y organizador para ambas: pedagogía y medicina se disputan el control de la infancia para sí, se disputan un lugar de enunciación privilegiado para imponer sus formas de nombrar y clasificar a la infancia; un poder que se imprime a la propia enunciación y ofrece así las condiciones para el despliegue de una red mucho más amplia de representaciones que decanta en prácticas concretas que signan la cotidianeidad. La medicina, erigida sobre un saber de base biológico con pretensiones de neutralidad y objetividad, demarca formas específicas de control sobre los cuerpos: estos se constituyen en sus máquinas-objeto de estudio de los cuales importa más lo que se puede observar y medir, que aquello que la experiencia subjetiva diga de él; configura en paralelo una noción de salud ideal, cercana a cierta perfección funcional establecida por parámetros, cantidades y concentraciones "normales", "correctas", estableciendo criterios a partir de los cuales cualquier desviación es considerada y tratada como patológica. Por su parte, la escuela, por medio de sus prácticas escolares y la imposición de ciertos modelos pedagógicos y lógicas de ordenamiento de los sujetos, pretende moldear a sus alumnos de modo homogéneo y, escudada bajo ciertos supuestos de calidad, capacidad, educabilidad, resultados, éxitos y fracasos, oculta el carácter ético-político del propio acto de educar, así como el entramado histórico y social desde el cual la educación asume su rol en la producción y reproducción cultural de una sociedad.

Plantear en estas condiciones la posibilidad de una escuela dentro del hospital, implica considerar críticamente esta maquinaria discursiva-institucional que las entrelaza de modo tan íntimo, en tanto instituciones con fuertes injerencias y miradas que imponen modos ser y habitar la infancia, modelos acabados de cómo construir con todo niño un alumno o un paciente, bajo mandatos que una y otra establecen en torno a su propio “deber ser" niño y, en ambos casos, recurriendo a una pretendida “justificación científica, moral y social de las técnicas de clasificación, identificación e intervención correctiva" (VALLEJOS, 2009, p.99) que les otorga un aire de verdad incuestionable. Tanto en un ámbito como en otro el cuerpo infantil es blanco de una inscripción disciplinaria que lo moldea según estos discursos hegemónicos normalizadores, restringiendo la potencia plena que emana del “devenir" infancia. Tal como afirmara Michel Foucault $(1979 ; 1992)$ nuestra sociedad ha desarrollado a la par del sistema de saber más complejo, las estructuras de poder más sofisticadas, ligando nuestras 
experiencias fundamentales, existenciales (la enfermedad, la muerte, el dolor, etc.) a dispositivos regulatorios, demarcatorios y clasificatorios que, sustentados en esos saberes, legitiman el uso (cierto uso) del poder. En sus palabras,

\begin{abstract}
(...) en cualquier sociedad, relaciones de poder múltiples atraviesan, caracterizan, constituyen el cuerpo social; y estas relaciones no pueden disociarse, ni establecerse, ni funcionar sin una producción, una acumulación, una circulación, un funcionamiento del discurso. No hay ejercicio del poder posible sin una cierta economía de los discursos de verdad que funcionen en, y a partir de esta pareja. (FOUCAULT, 1992, p.140)
\end{abstract}

En los últimos años, de la mano del crecimiento de los estudios y espacios de acción en torno a las infancias que intentan buscar miradas alternativas y de resistencia frente a estos discursos dominantes, se ha visibilizado un nuevo interés por la incorporación sistemática de escuelas, aulas y docentes en un espacio físico como el hospital. Sin ser ajenas a esta dimensión que entiende la escuela hospitalaria sujeta y atravesada por el fuerte cruce discursivo disciplinar médico-pedagógico, se viene poniendo énfasis en, por un lado, la posibilidad de poner en cuestión a partir de ella, estos discursos hegemónicos médico y pedagógico y sus principales supuestos, ampliando la discusión en torno a lo que el concepto de salud/enfermedad permite pensar y, por otro, instando a repensar al niño/a y sus necesidades, deseos y posibilidades más allá de la recuperación de sus condiciones fisiológicas, y a la infancia en situación de enfermedad como sujeto político y social pleno.

En esta línea, el reconocimiento del derecho a la educación se presenta como el fundamento principal de esta apuesta educativa. Sin embargo y más allá de los esfuerzos por ir construyendo nuevos sentidos en torno a estos espacios educativos, ser alumno en un hospital pediátrico, ser paciente en una escuela hospitalaria, parece significar aún en muchos casos, ser un pequeño rehén de las prácticas donde la mirada reduce la infancia a un "ser niño paciente-alumno" plenamente entregado a la construcción adulta de lo que ello significa, bajo una fuerte lógica de autoridad como ejercicio del poder que interviene y define la existencia de ese otro. Aún parece sostenerse el ritmo de un tiempo que desoye a los sujetos, que se olvida, que no logra ver, lo que puede una vida, lo que puede el pensamiento, lo que puede la infancia.

Frente a esto, la pregunta por los sentidos que se construyen alrededor de estas propuestas educativas no se agota en la respuesta por un derecho ganado. Sin desconocer el valor fundamental de esta dimensión, la ley como único argumento para justificar la presencia de la escuela en los hospitales no alcanza; responder sólo con argumentos que sostienen la palabra del derecho parece no 
bastar para justificar su existencia, parece dejar a la vista cierta incongruencia entre la voz jurídica y las "pasiones éticas” del educar (SKLIAR, 2011). Quizás porque todavía hoy se pierde de vista en ello las vidas singulares, las biografías particulares de estos niños reclamando su propia infancia; quizás por eso estos argumentos, al repetirse aisladamente, sin otras dimensiones de análisis (¿la filosófica, la ética, la política?) quedan desahuciados y vacíos, se vuelven insuficientes. Porque la pregunta que encarna la existencia del Otro en educación no se responde con más legislación, con más normativa, con más adaptaciones curriculares. Porque las preguntas que circulan en la escuela hospitalaria, las preguntas que nacen frente a la llegada y presencia de cada nuevo niño y su territorio de infancia, no encuentran respuesta ni en leyes, ni en ordenanzas, ni en didácticas. ¿Quién es ese Otro, ese alumno, ese/a niño/a que encontramos de frente a nosotros?, ¿cómo vive su dolor, cómo vive su padecer, cómo lo narra?, ¿qué es la educación para él?, ¿será acaso que algo de él y de su mundo se transformen mediando en ello el acto educativo, un vínculo, cierto encuentro, una relación construida en torno a lo educativo?

\section{PENSAR LO IMPOSIBLE: ESPACIOS-TIEMPOS DE RESISTENCIA EN LA ESCUELA}

Cierta incongruencia parece percibirse en aquellos primeros inicios de pensar lo educativo desde y en el hospital. Una incongruencia, sin embargo, nutrida de voces que tienen algo para decir, algo para preguntar, algo que rescatar de entre el gran edificio del discurso médico impuesto como irrevocable. La irrupción de la escuela en el ámbito hospitalario significa, sí, un cambio de mirada, cierta profanación de una lógica sedimentada, algo inesperado dispuesto a ocupar e inventar un nuevo espacio, y genera con ello fuertes cuestionamientos al discurso médico hegemónico, biologicista, pero también al núcleo duro del propio discurso pedagógico y sus supuestos. La escuela lentamente legitima su lugar dentro del hospital en tanto logra reinventar su modo de "hacer escuela" y pensarse en tanto tal. El desafío inicial supone no hacer de la escuela hospitalaria una escuela reproductora de un discurso biomédico, hablando en su propio lenguaje la lengua medicalizadora de la vida; el desafío se redoblaba si ello significa saltar, arrojarse a una experiencia que se pregunte cómo acoger a estos niños y niñas en sus situaciones particulares de enfermedad y dolor, y establecer relaciones educativas que permitan encontrar, construir y convergir en lo común del mundo y la singularidad de sus vida.

Encontrar los intersticios, los márgenes desde donde trastocar aquello que se impone como única posibilidad, para dar paso a otros modos de "hacer escuela", resulta tener más que ver con un “modo de estar" en estas escuelas, algo más que ver con la invención que con la repetición, con una afirmación comprometida ética y políticamente con el acto de educar. Porque implica dar una 
discusión sobre las fronteras entre posibilidad e imposibilidad, discutir qué es lo posible. En este sentido, afirmar que "lo posible no es lo que tenemos, no es 'lo que hay', sino lo que somos capaces de hacer en procura del futuro que imaginamos, en función de nuestras historias y de nuestro presente" (TERIGI, 2005, p.68), cobra una significación vital. Porque una discusión siempre reactualizable sobre qué es lo (im)posible en educación, parte del propio ejercicio de pensamiento que quiebra los límites entre lo posible y lo imposible, entre lo pensable y lo impensable, reconfigurando las fronteras de la creación y de la imaginación.

Quizás la potencia de la escuela hospitalaria siga siendo su posibilidad de dejar de pensar que es la enfermedad la que irrumpe en ella, o el contexto, o el paisaje hospitalario, o la lógica médica. Quien llega a estas escuelas es un Otro. Es la infancia. Un Otro, otra infancia y toda su existencia es la irrupción primera en una relación educativa para la que no se tienen respuestas o para la cual sólo se cuenta con respuestas prefabricadas a la medida de sujetos preexistentes que nunca son iguales a los que llegan a las aulas, sujetos construidos a fuerza de preconceptos, prejuicios y de un discurso normalizador ajustado a la disciplina escolar. Nuevamente el desafío: pensar al sujeto de la educación hospitalaria como alteridad inabarcable, sujeto “por-venir", sujeto en la educación y con posibilidades de ser otro a partir de ella. Y quizás la primera clave: el gesto de recibimiento, de bienvenida a un sujeto y su singular búsqueda de infancia como temporalidad, como modo de estar en el mundo.

\section{CORPORALIDADES Y SUBJETIVIDADES INFANTILES HABITANDO LOS ESPACIOS EDUCATIVOS.}

Somos un cuerpo, sí. No tenemos, apenas, un cuerpo. No sostenemos, apenas, un cuerpo. Somos un cuerpo desde la primera hora del día, hasta que lo que somos o creemos ser ya no puede más consigo mismo. Y aún así, seguimos siendo cuerpo (SKLIAR, 2011,

Somos un cuerpo, sí. Y somos también el modo de habitarlo y de ser parte del mundo por medio de él; somos en nuestros cuerpos y ellos son "lugares de existencia, y no hay existencia sin lugar, sin 'ahí', sin un 'aquí, 'he aquí'” (NANCY, 2010, p.16). Y en los encuentros educativos hospitalarios el cuerpo y la corporalidad, en tanto experiencia singular de ese cuerpo que habitamos y nos habita, se vuelven protagonistas en la escena educativa. Lo que sucede en cada cuerpo en tanto existencia, repercute en la forma de entender y relacionarnos con el mundo, de darle sentido a lo que en él sucede y a la presencia de cada quién en él.

Cuerpos sufrientes puestos frente a frente en cada encuentro educativo recorren las salas de hospital. Y el sufrimiento, que nos vuelve por igual cuerpos frágiles, vulnerables, es una de las experiencias comunes de los cuerpos infantiles en estas escuelas y aulas que irrumpen ante nosotros con sus marcas, con sus lenguajes... ¿Cómo pensar, entonces, un espacio para estos modos de 
vivenciar el cuerpo en estas escuelas?, ¿cómo pensar espacios para el mundo simbólico que representa el padecimiento, la enfermedad?, ¿cómo recibir a un sujeto que se constituye como tal en esas situaciones de enfermar, padecer, ser intervenido, ser invadido, y pretender sostener aún una escena educativa?

Es posible pensar una escuela hospitalaria dispuesta a ofrecer espacios de referencia para estos niños, escenarios desde los cuales repensarse en relación a su propia vivencia de dolor, de experimentar su cuerpo y de recuperar espacios de decisión, de elección; recuperar algo del propio deseo de ser parte de un mundo que excede las paredes del hospital. Escenarios donde estos niños y niñas vuelvan a ser y sentirse creadores de un mundo, cada quien un hacedor de un mundo compartido. Es posible imaginar una escuela capaz de ofrecer ese espacio tomando como primer gesto la hospitalidad. La hospitalidad como gesto educativo inicial. Porque la experiencia infantil de dolor, de enfermar, la cercanía de la muerte, no pueden entenderse y reducirse a parámetros fisiológicos, encerrarse en los límites de un cuerpo como objeto, tal como intenta presentarlo la biomedicina. Y la propuesta educativa que se piensa aquí, al entender esto, se presenta como un lugar de posibilidades a otras experiencias, como apuesta vital hacia una nueva noción de cuerpo en tanto representación construida y vivida, en tanto “cuerpos en relación, donde no exista ningún vestigio acerca de lo que falta o de lo que hace falta, de la idea del cuerpo normal, (...) de la obligación de juzgar" (SKLIAR, 2016, p.387), noción de cuerpo en tanto encuentro con el otro, “sin condiciones". Una educación hospitalaria capaz de ofrecer otros tiempos, otras narrativas, otras relaciones, distintas de las que la medicina dispone. Distintas de las que la pedagogía de la normalidad prescribe. Una educación signada por una respuesta ética como acompañamiento y recibimiento a una infancia plena. Una educación que ante todo ofrezca "gestos educativos" que tienen que ver, por un lado, con no reducir la infancia a la situación de enfermedad para predecir sus posibilidades o limitaciones en base a ella; por otro, con recuperar y dejar surgir el propio proyecto educativo y de vida de cada niño y niña, desde su propia voz, como palabra encarnada en una corporalidad singular. No se trata de negar el dolor, de silenciarlo, de intentar generar un espacio libre de tensión, de dificultades a la hora de encarar una tarea educativa. Pero se trata, sí, de desmarcar a cada niño sujeto de ese dolor como único soporte de su potencia o sus límites; de dejar de ver en su cuerpo sufriente la esencia de su existir, o los signos que sólo parecen conducir a un cuerpo en falta, en falla, incompleto, incorrecto y, en cambio, dar espacio al misterio de las infancias del cuerpo y del cuerpo de las infancias.

La potencia de estos gestos reside quizá en recrear la escuela como un espacio-tiempo que se abre a la existencia de cada niño/a, dejando nacer una relación que no sólo vea su cuerpo enfermo sino que converse con la corporalidad; que no sólo intente acercarse desde los modos preconcebidos 
de "formar" a cierto sujeto y entable, en cambio, una conversación con otros modos de subjetivación, con otros modos de "plegar" ese exterior compartido y volverlo subjetividad, conformación de un sujeto en situación, bajo la convicción de que "todo proceso de subjetivación no solamente conlleva nuevos campos de percepción y de afección, sino que constituye también él mismo una apertura de potencialidad" (DELEUZE, 2015, p.163).

Los cuerpos de estos niños dejan de ser apenas objeto de un discurso porque se transforma la experiencia hacia otras formas de relación con el cuerpo, entre cuerpos. Porque hay una construcción social, afectiva, responsiva por aquel otro como sujeto cuya existencia se sustenta, al tiempo que excede, su cuerpo, tornándola corporalidad vivida, experimentada, que se resiste al mandato disciplinador y normalizador. Ahí radica la potencia política de la relación educativa en este entorno hospitalario: en la construcción de una experiencia de existencia compartida, que a la vez ofrezca algo de un mundo común y transformable desde y para sí, generando un corrimiento de los límites construidos como imposibilidad.

Como afirma Cerletti (2008, p.16) "cada acto educativo actualiza un problema filosóficopolítico fundamental, que es cómo resolver la tensión entre reproducir lo que hay y dar un lugar diferente a lo que puede haber”. Y entonces, ¿qué hay de nuevo, de novedad en la educación hospitalaria?, ¿qué hay que no sea simple repetición de un modelo médico/escolar o un híbrido de ambos ceñido a modos de control del cuerpo y las subjetividades? Esto tiene que ver con cómo la propuesta educativa logra poner a disposición otros modos de ser sujeto en y de la educación, cómo ofrece un modo de relación particular con el mundo, con cómo lo educativo logra desplazarse hacia los márgenes de discursos y dispositivos médico-pedagógicos hegemónicos y, acaso, buscar otros modos de hablar, de actuar y de pensar la educación. Pero también tiene que ver con la posibilidad de una educación como espacio de libertad, donde el sujeto pueda, desee y decida expresarse en tanto tal, en su plena infancia como enigma, como aquello que irrumpe derribando certezas, estructuras y prejuicios. Implica, además, una propuesta capaz y dispuesta a esperar lo inesperado: porque donde impera por sobre toda otra posibilidad la cuantificación de supuestos logros educativos prefigurados, preconcebidos, es donde se desvanece la potencia del "por venir". Expresado de otro modo: "siempre que la educación se suponga productora de resultados, los docentes se verán forzados a trabajar con sus alumnos en una lógica productora de ciertos resultados. La pregunta real es ¿cómo crear un ambiente educativo donde lo inesperado sea valorado?” (BUTLER, 2017, p.176). Romper la lógica (re)productora de resultados, necesariamente, implica ser permeables a que lo inesperado nos movilice, ceder a la posibilidad de una afección mutua: una educación que afecta de modo singular a cada sujeto partiendo de un encuentro compartido de lenguajes hechos palabra y corporalidad; una 
afección mutua signada por la decisión de no abandonar la infancia tanto como no abandonar lo imposible como posibilidad; una afección que reconoce las formas de existencia infantil como modos de resistir frente a los mandatos externos deterministas.

Es cada niño ligado a su posibilidad de reconfiguración subjetiva quien encarna la sorpresa de un devenir sujeto, de una expresividad que es continuo comienzo, que se abre a la posibilidad de ser narrada como acontecimiento de existencia: es la posibilidad de "nacer la escuela" como experiencia infantil vitalmente ligada a lo nuevo, a la sorpresa, a los comienzos, a una infancia como sujeto de experiencia.

\section{CONSIDERACIONES FINALES: ¿QUÉ HAY DE HOSPITALARIO EN LAS ESCUELAS HOSPITALARIAS?}

Las escenas educativas hospitalarias son la base a partir de las cuales se afirma que hay otras subjetividades emergiendo aún de las situaciones más complejas en términos de sufrimiento y dolor. Las escenas educativas concretas, el "estar ahí" de docentes y alumnos, son el soporte desde el cual otras formas de pensar y cuestionar el acto educativo son posibles, son la potencia primera para reinventar sentidos, voces y miradas, y hacer protagonistas a quienes habían sido olvidados.

La pregunta por lo político de lo educativo tiene que ver con cómo encaramos los problemas considerados relevantes en determinados contextos escolares. Pensar qué propuesta educativa y qué sentidos crecen en las aulas hospitalarias, qué experiencias se está dispuesto a generar o a acompañar, son preguntas que se tornan propias del ámbito de lo político si se entiende que tales preguntas y sus respuestas, por precarias que sean, dan cuerpo a concepciones (sobre la enfermedad, sobre la infancia, sobre el sujeto en situación de enfermedad, etc.) que tienen consecuencias directas sobre las formas de mirar y de relacionarse con los sujetos de la educación en estos contextos, así como sobre los tiempos/espacios escolares posibles de pensar con y entre estos sujetos en tales situaciones.

En ese sentido, atreverse a asumir el desafío de pensar una educación hospitalaria a partir de experimentar y habitar ciertas prácticas escolares en hospitales, da cuenta de un modo particular de relación con el saber y de un modo también particular de acoger la infancia como otro existente más allá de las (im)posibilidades del dolor y la enfermedad. En cada acto educativo el compromiso con una mirada ética de respuesta por el otro se afirma al no abandonar a su suerte a cada niña y niño cuya singularidad se presenta inasible a causa de su dolor; al no condenarlos al silencio de un mundo que pareciera sólo posible y pensable como promesa por fuera de las paredes que delimita el hospital y, al contrario de ello, ofrecer y reconstruir mundos subjetivos en cada encuentro. De algún modo, se busca inaugurar una nueva narrativa de escuela, de sujeto, de infancia en el acontecimiento educativo. 
La sola irrupción en la escuela hospitalaria de las corporalidades y subjetividades de estos niños y niñas hospitalizados, que se expresan en lenguajes de dolor y sufrimiento, pero también de vitalidad plena, de celebración de la experiencia y la afectividad, impiden quedarse ajenos, insensibles a ellos y convocan a pensar otras formas de escuela, habitadas por otras formas de relación en ella. Aquello que nos deja perplejos, conmovidos, indefensos, es al mismo tiempo lo que permite preguntar y pensar desde otros espacios y tiempos, desde rincones de la propia forma de existir que iguala y singulariza en la fragilidad. Y, al mismo tiempo, reformula la pregunta por los sentidos del acto educativo en tanto relación entre sujetos y mundo. Estos niños y niñas, en sus modos de experimentar sus cuerpos, en sus modos de constituirse sujetos, al mismo tiempo que en su forma de ser parte de un encuentro educativo particular, parecen decir que algo de hospitalario vive y se construye en esas escuelas, en esas aulas, en esos encuentros en una sala, desde una cama. Un gesto de hospitalidad que acoge lo imposible, la relación con lo imposible, la incondicionalidad, que corre el riesgo que entraña lo imprevisto, lo inesperado, lo incalculable; que asume el riesgo de quedar expuestos, de ser vulnerables. Un gesto educativo de hospedar que sólo se vuelve tal en cuanto el acontecimiento se vuelve experiencia: se "hospeda" en el mismo momento en que se "hace" escuela.

La escuela hospitalaria se debate en esa marea que la lleva entre los destinos predeterminados por cierta suerte de "conjuro médico-educativo" y el inabarcable espacio abierto a la experiencia singular en una trama relacional común, en una experiencia como "modo de habitar el mundo de un ser que existe"(LARROSA, 2003, p.5).

Apostar a un espacio educativo, a la escuela inmersa en el contexto hospitalario, abre la posibilidad de ampliar los horizontes simbólicos de estos niños y niñas donde la enfermedad parece impregnarlo todo; abre el juego a otras experiencias posibles, más allá de las que la enfermedad clausura.

Ir al encuentro. Recibir y dejarnos recibir. Pensar el cuidado como acto político también propio del acto educativo. Conversar sobre el mundo, recrear el mundo, aún mirándolo y experimentándolo desde una cama. Responder ante otro aún sin tener respuestas posibles para el sufrir, el padecer, la finitud. Ser/hacer parte de una escuela hospitalaria, o hacer hospitalaria la escuela dentro del hospital, posiblemente tenga que ver, una vez más pensando con Skliar, no con actos heroicos, no con grandes transformaciones, sino con ciertos "gestos mínimos" para educar: "dar la bienvenida, saludar, acompañar, permitir, ser paciente, posibilitar, dejar, ceder, dar, mirar, leer, jugar, habilitar, atender, escuchar" (2011, p.263). Imaginar, inventar otros posibles encuentros. Hacer de lo imposible una posibilidad. Gestos educativos que sin duda debemos a las nuevas generaciones. 


\section{REFERENCIAS:}

BUTLER, J. (Re)Pensando la educación con Judith Butler: una cita necesaria entre filosofía y educación. En: GIULIANO, F. Rebeliones éticas, palabras comunes: conversaciones filosóficas, políticas, educativas. Buenos Aires: Miño y Dávila, 2017. p. 161-188.

CERLETTI, A. Repetición, novedad y sujeto en la educación: un enfoque filosófico y político. $1^{\text {a }}$ ed., Buenos Aires: Del Estante Editorial, 2008.

DELEUZE, G. El saber: curso sobre Foucault. Tomo I. 1ª ed., Buenos Aires: Cactus, 2013.

FOUCAULT, M. El orden del discurso. $2^{\text {a }}$ ed., Barcelona: Tusquets, 1979.

Microfísica del poder. $3^{\text {a }}$ ed., Madrid: Ediciones de la Piqueta, 1992.

LARROSA, J. La experiencia $\mathbf{y}$ sus lenguajes. Disponible en: <http://www.me.gov.ar/curriform/publica/oei_20031128/ponencia_larrosa.pdf>. Acceso: 19 jun. 2017.

NANCY, J. L. Corpus. $2^{a}$ ed., Madrid: Arena Libros, 2010.

SKLIAR, C. El lenguaje de la norma y los individuos frágiles. Childhood\&Philosophy. Río de Janeiro, v.12, n.24, ago. 2016, p. 371-389. Disponible en: 〈http://www.e-publicacoes.uerj.br/index.php/childhood/article/view/22964〉. Acceso: 24 jun. 2017.

Lo dicho, lo escrito, lo ignorado: ensayos mínimos entre educación, filosofía y literatura. $1^{\mathrm{a}}$ ed., Buenos Aires: Miño y Dávila, 2011.

TERIGI, F. Collected papers: sobre las decisiones en el gobierno de la educación. En: FRIGERIO, G.; DIKER, G. (Comps.). Educar: ese acto político. Buenos Aires: Del Estante Editorial, 2005. p. 63-73.

VALLEJOS, I. La categoría de normalidad: una mirada sobre viejas y nuevas formas de disciplinamiento social. En: ROSATO, A.; ANGELINO, MA. (Coords.) Discapacidad e ideología de la normalidad: desnaturalizar el déficit. Buenos Aires: Noveduc, 2009. p. 95-115. 


\section{CORPORALIDADES, TEMPORALIDADES E INFANCIAS: LA PREGUNTA POR LA HOSPITALIDAD DESDE LA ESCUELA}

Resumen. Las escuelas hospitalarias son espacios educativos que permiten la continuidad escolar de niños hospitalizados en situación de enfermedad. En estos ámbitos los discursos médico y pedagógico hegemónicos se entrelazan bajo un eje común normalizador propio de dispositivos destinados al control de los cuerpos y las subjetividades, obturando posibilidades propias de la existencia infantil. Se propone aquí problematizar estos discursos y pensar posibles intersticios donde lo educativo pueda desafiar los poderes y saberes dominantes. Se busca pensar nuevos modos de encuentro y relación educativa partiendo de la hospitalidad como reconocimiento y acogida de otras posibles corporalidades y temporalidades infantiles, como gesto de resistencia, transformación y afección mutua que habite la escuela.

Palabras clave: Escuelas hospitalarias. Hospitalidad. Corporalidades.

\section{CORPORALITIES, TEMPORALITIES AND CHILDHOOD: THE QUESTION FOR HOSPITALITY FROM SCHOOL}

Abstract. The hospital schools are educative spaces which allow that hospitalized children continue with their scholar careers. In those ambits the hegemonic language of medicine and pedagogy merge into one common axis typical of devices which are destined to control the bodies and the subjectivities, and therefore impeding the intrinsic possibilities of childhood. The proposal is to discuss these wordings and to think about possible interstices where the education could challenge the powers and dominant theories. The goal is to search for new kinds of encounter and educational relationship starting from hospitality as a source and acknowledgement of other possible childhood's corporalities and temporalities, as a gesture of resistance, transformation and mutual affections to inhabit the schools.

Keywords: Hospital schools. Hospitality. Corporalities.

\section{CORPORALIDADES, TEMPORALIDADES E INFÂNCIAS: A PERGUNTA PELA HOSPITALIDADE DESDE A ESCOLA}

Resumo. As escolas hospitalares são espaços educativos que permitem a continuidade escolar das crianças doentes e hospitalizadas. Nesses âmbitos os discursos médico e pedagógico hegemônicos entrelaçam-se sob um eixo comum normalizador próprio dos dispositivos de controle dos corpos e subjetividades, restringindo as possibilidades próprias da existência infantil. Propõe-se aqui problematizar esses discursos e pensar possíveis interstícios onde o educativo possa desafiar os poderes e saberes dominantes. Procura-se pensar novos modos de encontro e relações educativas partindo da hospitalidade como reconhecimento e acolhida para outras possíveis corporalidades e temporalidades infantis, como gesto de resistência, transformação e afecção mútua para habitar na escola.

Palavras-chave: Escolas hospitalares. Hospitalidade. Corporalidades. 